\title{
Comparision effects of solifenacin, darifenacin, propiverine on ocular parameters in eyes: A prospective study
}

\author{
Mahmut Taha Ölçücü ${ }^{1}$, Kerem Teke ${ }^{1}$, Kadir Yildirim ${ }^{2}$, Mesut Toğaç ${ }^{3}$, Burcu Işık ${ }^{3}$, Yusuf Cem Yilmaz ${ }^{3}$ \\ ${ }^{1}$ Department of Urology, Agri State Hospital, Agri, Turkey; ${ }^{2}$ Department of Urology, Elaziğ Education \\ and Research Hospital, Elazig, Turkey; ${ }^{3}$ Department of Ophthalmology, Agri State Hospital, Agri, Turkey
}

\section{ABSTRACT}

Objective: To evaluate the effects of solifenacin, darifenacin, and propiverine on nasal-, subfoveal-, temporal choroidal thicknesses (NCT, SFCT, TCT), intraocular pressure (IOP) and pupil diameter (PD).

Materials and Methods: Patients with overactive bladder (OAB) diagnosed according to The International Continence Society were administered with solifenacin, darifenacin or propiverine on a daily basis between November 2017 and May 2018. NCT, SFCT, TCT, IOP, and PD of these patients were measured and compared as initial, fourth and twelfth weeks.

Results: A total of 165 patients (330 eyes) with OAB were evaluated. Solifenacin $(\mathrm{n}=140)$ significantly reduced IOP from $17.30_{ \pm} .72 \mathrm{mmHg}$ to $16.67 \pm 2.56 \mathrm{mmHg}$ $(\mathrm{p}=0.006)$ and $16.57 \pm 2.41 \mathrm{mmHg}(\mathrm{p}=0.002)$, at the fourth and twelfth weeks, respectively. Darifenacin $(n=110)$ significantly reduced NCT from $258.70 \pm 23.96 \mu \mathrm{m}$ to $257.51 \pm 22.66 \mu \mathrm{m}(\mathrm{p}=0.002)$ and $255.36 \pm 19.69 \mu \mathrm{m}(\mathrm{p}=0.038)$, at the fourth and twelfth weeks, respectively. Propiverine $(n=80)$ significantly increased PD from $4.04 \pm 0.48 \mathrm{~mm}$ to $4.08 \pm 0.44 \mathrm{~mm}(\mathrm{p}=0.009)$ and $4.09 \pm 0.45 \mathrm{~mm}(\mathrm{p}=0.001)$, at the fourth and twelfth weeks, respectively.

Conclusion: These findings can help to decide appropriate anticholinergic drug choice in $\mathrm{OAB}$ patients. We finally suggest further well-designed randomized prospective studies with a larger population to evaluate the anticholinergic-related complications in eyes.

\section{ARTICLE INFO}

(D) Mahmut Taha Ölçü̈c̈̈
http://orcid.org/0000-0002-4721-2807

Keywords:

Pressure; Urinary Bladder;

Overactive; Anisocoria

Int Braz J Urol. 2020; 46: 185-93

Submitted for publication:

February 08, 2019

Accepted after revision:

October 13, 2019

Published as Ahead of Print: November 30, 2019

\section{INTRODUCTION}

Both sexes are affected by an overactive bladder $(\mathrm{OAB})$, which is a common disorder with a reported prevalence between $11.8 \%$ and $16.9 \%$. Symptoms of $\mathrm{OAB}$ include urgency, frequent urination, and nocturia with or without urge incontinence (1-3). Lifestyle modifications and behavioral therapies are the first steps taken into treatment, however, medical therapy constitutes the mainstay method (4). Oral anticholinergics are the first-line drugs in medical treatment (5). Due to various settlements of cholinergic (muscarinic) receptors (M1-M5) in the body, the side effects of anticholinergics emerge in accordance with this (6). These drugs can cause side effects such as dry mouth, constipation, nausea, vomiting, headache, and confusion $(7,8)$.

There are extensive $\mathrm{M}$ receptors in iris sphincter muscle, ciliary muscle and trabecular 
cells (9-11). Iris sphincter muscle and ciliary muscles are relevant with accommodation and changing of pupil diameter (PD) $(12,13)$. M3 receptors are most common cholinergic receptors than the other types in iris sphincter and ciliary muscle cells $(10,14)$. Trabecular cells control intraocular pressure (IOP) by changing the aqueous humor drainage (15). Blockage of these receptors may lead to blurred vision, dry eyes, and increased IOP in angle-closure glaucoma $(16,17)$. Choroid is one of the most vascularised areas in the body, allowing outer retina to become vascularised (18). Increased choroidal thickness (CT) can cause haemorrhage and exudation. Reducing this thickness can decrease blood flow of retina (18). Age-specific (haemorrhagic and exudated) choroidal thickening is known to be a poor prognostic factor in age-related macular degeneration. It is reported that choroidal layer contains smooth muscles with intense cholinergic innervations $(18,19)$.

In this context, since anticholinergic drugs may affect ocular $M$ receptors, we herein aimed to evaluate the effects of solifenacin, darifenacin, and propiverine on nasal-, subfoveal-, temporal CTs (NCT, SFCT, TCT), IOP and PD in the eyes.

\section{MATERIALS AND METHODS}

The study was designed prospectively non-randomized in accordance with the Helsinki Declaration after approval of Erzurum Atatürk University Medical Faculty Ethics Committee (Approval Number: B.30.2.ATA.0.01.00/128). Patients who were diagnosed with $\mathrm{OAB}$ in urology clinic between November 2017 and May 2018 were included and referred to ophthalmologic clinic after receiving the informed consents from patients. The diagnosis of $\mathrm{OAB}$ was made according to the description described earlier by the International Continence Society (ICS) (20). Informed consent forms were obtained from patients who wanted to participate in the study. The costs of the drugs were paid by the patient's own health insurance. As a result of detailed urological and ophthalmological evaluations, patients with histories of any systemic diseases (e.g. diabetes mellitus, hypertension, coronary artery disease), renal insufficiency, urinary tract infections, previous use of anticho- linergic agents, angle-closure glaucoma, ocular trauma, ocular surgery, high refractive error $(> \pm 6 \mathrm{D})$, intraocular drug injection, photodynamic or photocoagulation laser treatments, use of topical ophthalmologic drops, pathology in ophthalmologic examinations and refusing follow-ups or discontinue the medicines for any reason were excluded from the study (Figure-1). No patient gave up on $\mathrm{OAB}$ treatment because of ocular findings or ocular side effects.

Patients with $\mathrm{OAB}$ were administered with $5 \mathrm{mg}$ solifenacin $1 \times 1,7.5 \mathrm{mg}$ darifenacin $1 \times 1$ or $30 \mathrm{mg}$ sustained-release propiverine $1 \mathrm{x} 1$ on a daily basis. When we analyzed retrospectively, we found that the most preferred anticholinergics were solifenacin, darifenacin, propiverine, oxybutynin, and trospium, respectively in our clinic. Hence we aimed to compare the eyes of patients who were treated with most preferred three anticholinergics (solifenacin, darifenacin, propiverine) for $\mathrm{OAB}$ treatment. As known, the effects of anticholinergics are similar. But more adverse effects can be observed with some anticholinergics than others. Two urologists (MTO and KT) distributed the anticholinergics in a regular order to the patients with $\mathrm{OAB}$. We did not make any randomization in this study. We tried to keep equally the number of groups. Some factors affected the number of participants who have finished the study among groups. Those factors included: patients who gave up the anticholinergic because of adverse effects, did not come to follow-up, and were discordant throughout treatment. In method, we have specified those factors as the excluded criteria described above. We observed more adverse effects in propiverine group. And it affected the number of propiverine group. The ophthalmologists who examined the patients did not know which anticholinergics were used by the patients. SFCT, NCT, TCT, IOPs, and PDs in eyes of the patients in the fourth and twelfth weeks were evaluated by ophthalmologists. CT was measured at 500-micron intervals of the section from $1 \mathrm{~mm}$ nasal and temporal to the subfoveal center via an optical coherence tomography device (Topcon 3d 0ct-2000 Fa Plus, Topcon Inc., Japan). IOP was measured with a computerized tonometer (Topcon Ct-800, Topcon Inc., Japan), 
Figure 1 - Flow-chart of the our prospective cohort study.

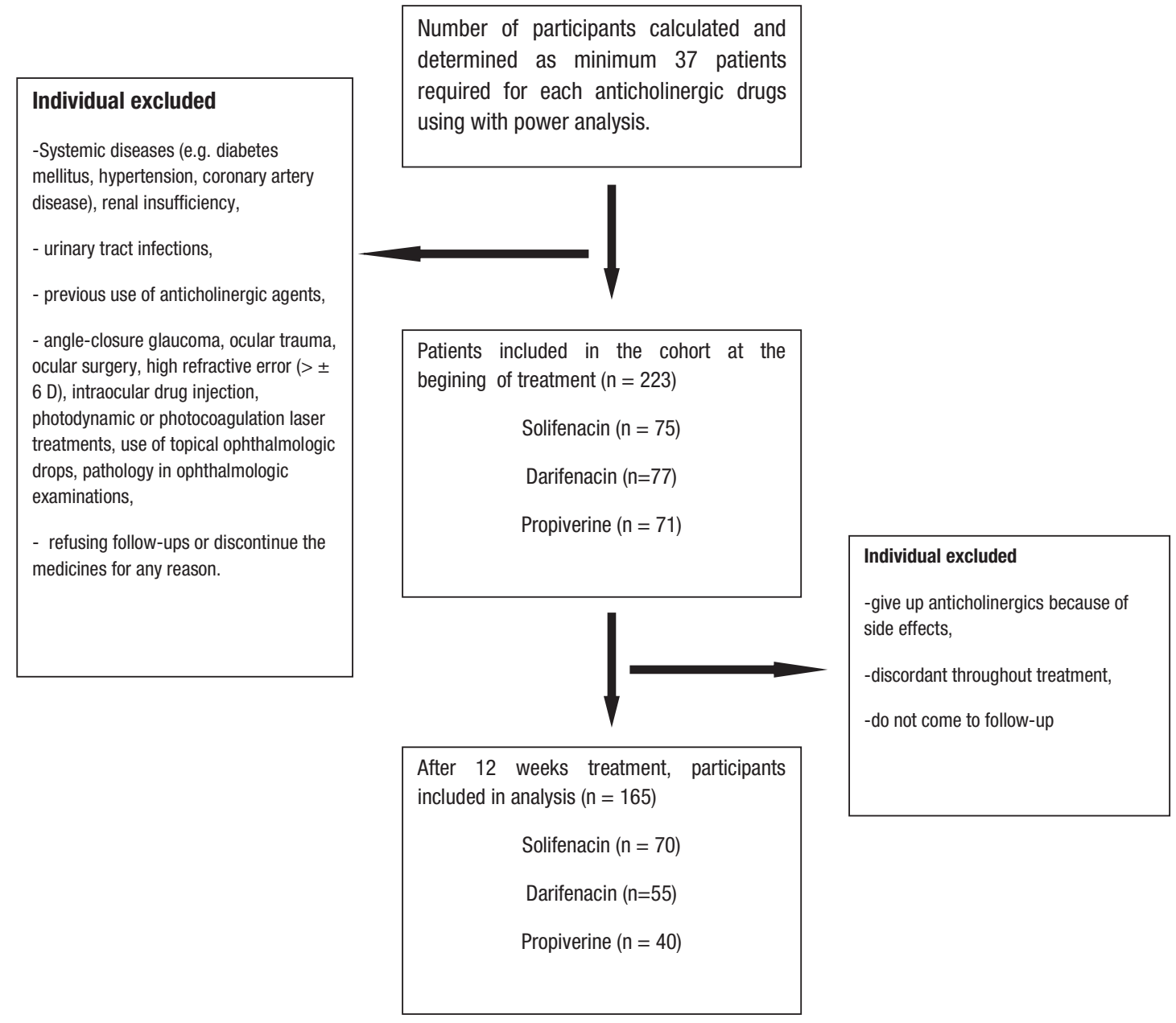

and PD was measured by an autorefractometer (Topcon Kr-800, Topcon Inc., Japan).

When we assigned the statistical power as 0.8 , the alpha as 0.05 , effect size as 0.3 and group size as 3 the power analysis has shown minimum required sample size as 111 . So a minimum of 37 patients were required for each anticholinergic drug. The mean values of the measurements for both eyes were recorded as data for each patient. The results were statistically analyzed with SPSS 15.0. Normality test was used to determine the pretreatment measurements among solifenacin, darifenacin, propiverine groups. Due to the data is normally distributed, ANOVA test was used to compare independent variables regarding those three groups. For the ages of the three groups, the
Kruskal Wallis test was used after the descriptive statistics, and two samples paired t-test was used to compare the values measured in the fourth and twelfth weeks for the same individuals depending on the medicines used. A value of $p<0.05$ was deemed statistically significant.

\section{RESULTS}

One hundred sixty-five patients (330 eyes) who have completed 12 weeks follow-up received solifenacin $(n=70)$, darifenacin $(n=55)$, and propiverine $(\mathrm{n}=40)$ (Figure-1). A hundred eighteen $(71.5 \%)$ patients were female and $47(28.5 \%)$ were male. Female and male ratio for the solifenacin, darifenacin and propiverine groups were 59 
(84.3\%) vs. $11(15.7 \%), 30(54.5 \%)$ vs. 25 (45.5\%), and $29(72.5 \%)$ vs. $11(27.5 \%)$, respectively. Total mean age was $50.10 \pm 13.17$ years (ranging from 19 to 81 ) and observed as $49.37 \pm 13.17$ (ranging from 19 to 76 ) in the solifenacin group, as $50.49 \pm 12.50$ (ranging from 21 to 75 ) in the darifenacin group, and as $50.87 \pm 14.31$ (ranging from 29 to 81 ) in the propiverine group. There was no significant difference between the mean age of the groups $(p=0.848)$. No statistically differences were determined between baseline pretreatment measurement of SCFT $(p=0.587)$, NCT $(p=0.430)$, TCT $(p=0.365)$ and PD $(p=0.960)$ among solifenacin, darifenacin, propiverine groups. However, baseline pretreatment values of IOP $(p=0.010)$ were statistically difference among those three groups (Table-1).

\section{Choroidal Thickness}

Subfoveal Choroidal Thickness: No statistically significant differences were observed be- tween the pretreatment and fourth week or between the pretreatment and the twelfth week for SCFT in the solifenacin-, darifenacin-, and propiverine-treated groups ( $p>0.05$ ) (Table-2). Nasal Choroidal Thickness: The mean value of NCT slightly but statistically increased at fourth week in solifenacin group $(p=0.042)$.. However, rising NCT regressed to approximately pretreatment levels at the twelfth week in these patients $(p=0.849)$. The darifenacin treatment gradually reduced the mean of NCT at the fourth and twelfth weeks compared to pretreatment levels. These decreases were significantly different at the fourth $(\mathrm{p}=0.002)$ and twelfth weeks $(p=0.038)$ according to the pretreatment baseline. In propiverine group, the mean value of NCT was significantly reduced by the fourth week $(\mathrm{p}=0.012)$. At the twelfth week, NCT had increased and reached approximately pretreatment levels ( $p=0.438)$ (Table-2). Temporal Choroidal Thickness: In all three treatment groups (solifenacin, darifenacin, and propiverine), there was no statistically

Table 1 - The pretreatment results of descriptive analysis regarding to three anticholinergic groups are presented.

\begin{tabular}{|c|c|c|c|c|}
\hline & $\begin{array}{l}\text { Solifenacin } \\
\qquad(\mathrm{n}=70)\end{array}$ & $\begin{array}{l}\text { Darifenacin } \\
\qquad(\mathrm{n}=55)\end{array}$ & $\begin{array}{l}\text { Propiverine } \\
\quad(\mathrm{n}=40)\end{array}$ & $P$ \\
\hline \multicolumn{5}{|l|}{ Gender } \\
\hline Female $(\%)$ & $59(84.5 \%)$ & $30(54.5 \%)$ & $29(72.5 \%)$ & - \\
\hline Male $(\%)$ & $11(15.5 \%)$ & $25(45.5 \%)$ & $11(27.5 \%)$ & \\
\hline Age (mean $\pm S D)$ & $49.37 \pm 13.17$ & $50.49 \pm 12.50$ & $50.87 \pm 14.31$ & 0.848 \\
\hline $\begin{array}{l}\text { Subfoveal choroidal thickness }(\mu \mathrm{m}) \\
\text { (mean } \pm \text { SD) }\end{array}$ & $253.52 \pm 26.72$ & $258.66 \pm 33.08$ & $258.80 \pm 38.08$ & 0.587 \\
\hline $\begin{array}{l}\text { Nasal choroidal thickness }(\mu \mathrm{m}) \text { (mean } \pm \\
\text { SD) }\end{array}$ & $252.48 \pm 24.64$ & $258.70 \pm 23.96$ & $256.17 \pm 33.34$ & 0.430 \\
\hline $\begin{array}{l}\text { Temporal choroidal thickness }(\mu \mathrm{m}) \text { (mean } \\
\pm \mathrm{SD} \text { ) }\end{array}$ & $251.30 \pm 29.43$ & $258.08 \pm 22.98$ & $251.47 \pm 33.53$ & 0.365 \\
\hline $\begin{array}{l}\text { Intraocular pressure }(\mathrm{mmHg}) \text { (mean } \pm \\
\mathrm{SD})\end{array}$ & $17.30 \pm 2.72$ & $17.05 \pm 3.40$ & $15.50 \pm 3.02$ & $0.010^{*}$ \\
\hline Pupil diameter $(\mathrm{mm})($ mean $\pm \mathrm{SD})$ & $4.06 \pm 0.53$ & $4.03 \pm 0.43$ & $4.04 \pm 0.48$ & 0.960 \\
\hline
\end{tabular}




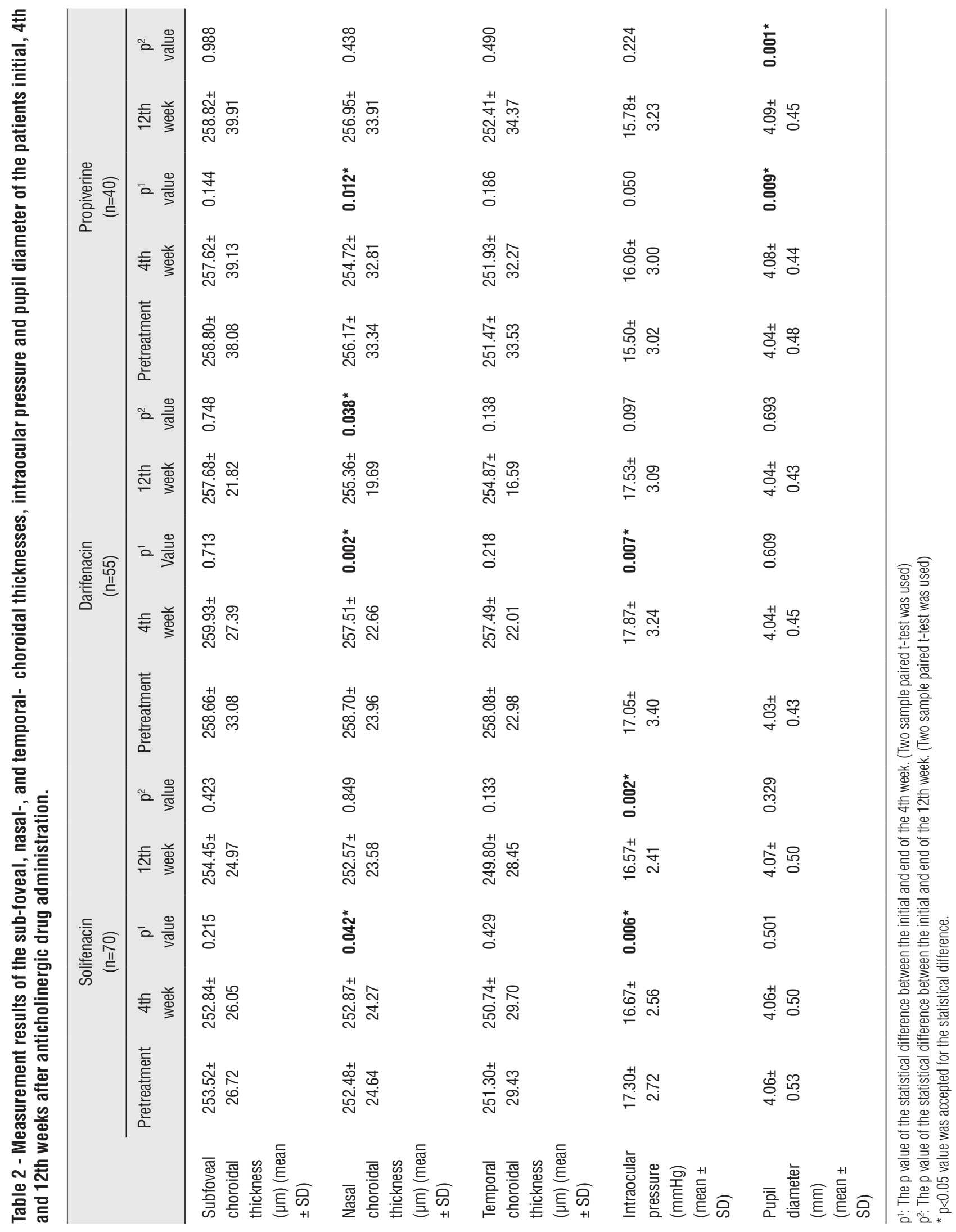


significant difference in the fourth and twelfth weeks when compared to the pretreatment period $(p>0.05)$ (Table-2).

\section{Intraocular Pressure}

Four and twelve weeks after solifenacin treatment, when compared to the mean level of pretreatment, the mean values of IOP were significantly reduced ( $p=0.006$ and $p=0.002$, respectively). The results at four weeks after darifenacin treatment showed a significant increase in mean IOP when compared to the mean pretreatment level $(p=0.007)$. The mean IOP level in patients treated with darifenacin after twelve weeks was lower than after the four-week treatment period, and it also did not have any statistically significant difference when compared with the pretreatment IOP levels $(p=0.097)$. In propiverine group, the IOP changes were not found to be statistically significant when compared to the pretreatment levels at forth and twelfth weeks ( $p$ >0.05) (Table-2).

\section{Pupil Diameter}

The changes of PD with solifenacin and darifenacin, according to pretreatment levels, were not significantly different at fourth and twelfth weeks ( $p>0.05)$. Unlike solifenacin and darifenacin, propiverine treatment significantly increased PD at the fourth $(p=0.009)$ and twelfth weeks $(p=0.001)$ when compared to pretreatment levels (Table-2).

\section{DISCUSSION}

M2 receptors are the most intensive muscarinic receptor types in the bladder and mostly M2 and M3 receptors play a role in $\mathrm{OAB}$ physiopathology (21). It is known that darifenacin and solifenacin are more selective for M3 than M2 receptors (6). Propiverine, oxybutynin, fesoterodine, and trospium are nonselective anticholinergics $(22,23)$. It has also been reported propiverine serves as a calcium channel blocker and increases relaxation in smooth muscles, making it more efficient in treating symptoms of OAB (23). In studies conducted by evaluating the effects of systemic anticholinergics on eyes, it has been emphasized that attention should be paid to the use of these medicines in patients with ophthalmologic diseases, especially dry eye and angle-closure glaucoma $(24,25)$. We considered these suggestions when choosing criteria for patient's inclusion in the study.

Telek et al. studied $61 \mathrm{OAB}$ patients and the effects of three months of $1 \times 1$ daily oral administration of sustained-release tolterodine on SFCT, NCT, TCT, IOP, and PD (7). When the values before and after treatment and the p-values were compared, it was observed that SFCT $(p=0.862)$ NCT $(p=0.658)$, TCT $(p=0.497)$, IOP ( $p=0.732)$, PD ( $p=0.711)$ had no differences. The results demonstrated that there was no statistically significant difference on the parameters before and after treatment. However, it was stated that these findings should be supported by other studies.

Turkoglu et al. evaluated daily $1 \times 1$ administration of oral trospium to 80 female $\mathrm{OAB}$ patients and IOP changes before treatment as well as in the fourth and twelfth weeks of treatment. No significant difference was found in the study for IOP changes in the fourth and twelfth weeks compared to pretreatment $(p=0.251$, $p=0.340$, respectively), however, it was reported that trospium significantly reduced tear secretion (26). Likewise, in another study conducted by Turkoglu et al. in 2015, no significant difference was observed in IOP values at commencement, the fourth week, or the twelfth week of daily $1 \times 1$ oral solifenacin in 93 female $O A B$ patients (27). It was also emphasized in this study that solifenacin did not affect tear secretion. Sekeroglu et al. compared the IOPs of 60 female $\mathrm{OAB}$ patients who used solifenacin for four weeks and a control group of 30 healthy females at commencement and four weeks (28). They found no significant difference in either group ( $p=0.864$ and $p=0.160$, respectively), and, hence, they reported solifenacin does not affect IOP. In these two studies, effect of solifenacin on CT, IOP, and PD is not statistically significant. However, in these studies, it was observed that solifenacin decreased IOP even though it was not statistically significant in some individuals. In our study, we concluded that solife- 
nacin significantly decreased IOP at the fourth week, and this decline was more pronounced in the twelfth week. Even though IOP significantly decreased in those who used solifenacin, it remained within normal limits $(9-22 \mathrm{mmHg})$ in our study.

Altan-Yaycioglu et al. conducted a study on 52 OAB patients, administering oral tolterodine to 28 of them and oxybutynin to remaining 24. The authors then compared the patient's IOPs and PDs with the initial values at the four-week follow-up. No significant difference was found among the initial values except for PD in dark light in the group that used tolterodine ( 3.72 vs. $4.16 \mathrm{~mm}, \mathrm{p}=0.025$ ) (29). In this study, neither agent affected tear secretion.

Although it is generally recommended that anticholinergics should not be used in $\mathrm{OAB}$ patients with angle-closure glaucoma, it was concluded by a randomized, double-blind, placebo-controlled study conducted by Gatchev et al. in 2016 that propiverine does not change IOP in patients with angle-closure glaucoma (30). This study suggested that propiverine could increase PD in patients with wide-angle and angle-closure glaucoma compared to a placebo (28). In our study, we concluded that propiverine did not alter IOP and significantly increased PD at the fourth week and twelfth week.

An important finding of our study is the effect of darifenacin on NCT. According to the results we obtained, usage of darifenacin for twelve weeks progressively decreases NCT. It is reported that the perfusion pressure is important for blood supply to this area (31). Therefore, factors that might spoil ocular blood flow may lead to decreased NCT (32). However, the reason for this finding should be investigated in more detail.

Furthermore, there was a statistically significant difference in the fourth week, and some parameters that improved in the twelfth week also attracted attention in our study. NCT significantly increased and then returned to normal levels for those who used solifenacin, IOP significantly increased and then returned to normal levels for those who used darifenacin, and NCT significantly decreased and then returned to normal levels for those who used propiverine. Perhaps this situation may be related to some adaptation mechanisms in the eye.

This work has several limitations. A major lack of this study is its non-randomized design and the fact that the groups were not evenly distributed. Additionally, even if some baseline characteristics like CT and PD of treatments groups were homogenously distributed, pretreatment IOP from three groups was statistically different. Another notable limitation, anticholinergic-related complications like dry mouth, constipation, and confusion were not mentioned in this cohort. Further works are needed to evaluate the anticholinergic-related complications in collaboration with some parameters for eye and to compare the treatment groups, which have similar baseline characteristics.

In conclusion, solifenacin significantly reduced IOP, darifenacin significantly reduced NCT and propiverine significantly increased PD in patients with $\mathrm{OAB}$ who had normal ophthalmologic examinations after the twelve weeks of treatment. These findings can help to decide appropriate anticholinergic drug choice in $\mathrm{OAB}$ medical treatment for patients with eye-related disorders. We suggest these findings should be supported by further well-designed randomized prospective studies with larger populations for better reliability.

\section{ACKNOWLEDGEMENTS}

Thanks to Sezai Ustun AYDIN (MD) for his support to improve the language of the manuscript.

Ethics Committee Approval: Ethics committee approval was received for this study from the ethics committee of Erzurum Ataturk University Medical Faculty

\section{CONFLICT OF INTEREST}

None declared. 


\section{REFERENCES}

1. Stewart WF, Van Rooyen JB, Cundiff GW, Abrams P, Herzog $A R$, Corey $R$, et al. Prevalence and burden of overactive bladder in the United States. World J Urol. 2003;20:327-36.

2. Irwin DE, Milsom I, Kopp Z, Abrams P, Artibani W, Herschorn S. Prevalence, severity, and symptom bother of lower urinary tract symptoms among men in the EPIC study: impact of overactive bladder. Eur Urol. 2009;56:14-20.

3. Zumrutbas AE, Bozkurt Al, Tas E, Acar Cl, Alkis 0, Coban K, et al. Prevalence of lower urinary tract symptoms, overactive bladder and urinary incontinence in western Turkey: results of a population-based survey. Int J Urol. 2014;21:1027-33.

4. Dhaliwal P, Wagg A. Overactive bladder: strategies to ensure treatment compliance and adherence. Clin Interv Aging. 2016;11:755-60.

5. Andersson KE. Antimuscarinics for treatment of overactive bladder. Lancet Neurol. 2004;3:46-53.

6. Abrams P, Andersson KE. Muscarinic receptor antagonists for overactive bladder. BJU Int. 2007;100:987-1006.

7. Telek HH, Doluoglu OG, Burcu A, Demirok G, Ornek F, Ayyildiz $A$. The effects of tolterodine on anterior segment and choroidal thickness in patients with overactive bladder syndrome. Ther Adv Urol. 2017:9:91-7.

8. Chapple CR, Khullar V, Gabriel Z, Muston D, Bitoun CE, Weinstein D. The effects of antimuscarinic treatments in overactive bladder: an update of a systematic review and meta-analysis. Eur Urol. 2008;54:543-62.

9. Gupta N, Drance SM, McAllister R, Prasad S, Rootman J, Cynader MS. Localization of M3 muscarinic receptor subtype and mRNA in the human eye. Ophthalmic Res. 1994;26:207-13.

10. Woldemussie E, Feldmann BJ, Chen J. Characterization of muscarinic receptors in cultured human iris sphincter and ciliary smooth muscle cells. Exp Eye Res. 1993;56:385-92.

11. Zhang $X$, Hernandez MR, Yang H, Erickson K. Expression of muscarinic receptor subtype mRNA in the human ciliary muscle. Invest Ophthalmol Vis Sci. 1995;36:1645-57.

12. Newsome DA, Loewenfeld IE. Iris mechanics. II. Influence of pupil size on details of iris structure. Am J Ophthalmol. 1971;71:553-73.

13. Koretz JF, Kaufman PL, Neider MW, Goeckner PA. Accommodation and presbyopia in the human eye--aging of the anterior segment. Vision Res. 1989;29:1685-92.

14. Gil DW, Krauss HA, Bogardus AM, WoldeMussie E. Muscarinic receptor subtypes in human iris-ciliary body measured by immunoprecipitation. Invest Ophthalmol Vis Sci. 1997;38:1434-42.
15. Selbach JM, Gottanka J, Wittmann M, Lütjen-Drecoll E. Efferent and afferent innervation of primate trabecular meshwork and scleral spur. Invest Ophthalmol Vis Sci. 2000;41:2184-91.

16. Ruxton K, Woodman RJ, Mangoni AA. Drugs with anticholinergic effects and cognitive impairment, falls and all-cause mortality in older adults: A systematic review and meta-analysis. Br J Clin Pharmacol. 2015;80:209-20. Erratum in: Br J Clin Pharmacol. 2015;80:921-6.

17. Mohammed ZS, Simi ZU, Tariq SM, Ali KR. Bilateral acute angle closure glaucoma in a 50 year old female after oral administration of flavoxate. Br J Clin Pharmacol. 2008;66:726-7.

18. Nickla DL, Wallman J. The multifunctional choroid. Prog Retin Eye Res. 2010;29:144-68.

19. Meriney SD, Pilar G. Cholinergic innervation of the smooth muscle cells in the choroid coat of the chick eye and its development. J Neurosci. 1987;7:3827-39.

20. Haylen BT, de Ridder D, Freeman RM, Swift SE, Berghmans $B$, Lee J, et al. An International Urogynecological Association (IUGA)/International Continence Society (ICS) joint report on the terminology for female pelvic floor dysfunction. Int Urogynecol J. 2010;21:5-26.

21. Gupta K, Kaushal S. Medical treatment of overactive bladder: an overview. Curr Clin Pharmacol. 2012;7:229-39.

22. Marcelissen T, Rashid T, Antunes Lopes T, Delongchamps NB, Geavlete B, et al. Oral Pharmacologic Management of Overactive Bladder Syndrome: Where Do We Stand? Eur Urol Focus. 2019;5:1112-9.

23. Hopkinson V, Pearce I. Pharmacotherapy in overactive bladder. Journal of Clinical Urology 2018;11:38-47.

24. Sekeroglu MA, Hekimoglu E, Tasci Y, Dolen I, Arslan U. Ocular surface changes following oral anticholinergic use for overactive bladder. Cutan Ocul Toxicol. 2016;35:218-21.

25. Ozen Tunay Z, Ozdemir O, Ergintürk Acar D, Cavkaytar S, Ersoy E. Dry eye findings worsen with anticholinergic therapy in patients with urge incontinence. Int Urogynecol J. 2016;27:919-22.

26. Turkoglu AR, Parmak Yener N, Coban S, Guzelsoy M, Emul A, Demirbas M, et al. Effect of trospium chloride therapy on intraocular pressure and tear secretion in overactive bladder patients. Cutan Ocul Toxicol. 2017;36:331-5.

27. Turkoglu AR, Yener NP, Coban S, Guzelsoy M, Demirbas $M$, Demirci $H$. Changes in intraocular pressure and tear secretion in patients given $5 \mathrm{mg}$ solifenacin for the treatment of overactive bladder. Int Urogynecol J. 2017;28:777-81.

28. Sekeroglu MA, Hekimoglu E, Petricli IS, Tasci Y, Dolen I, Arslan U. The effect of oral solifenacin succinate treatment on intraocular pressure: glaucoma paradox during overactive bladder treatment. Int Urogynecol J. 2014;25:1479-82. 
29. Altan-Yaycioglu R, Yaycioglu O, Aydin Akova Y, Guvel $S$, Ozkardes $H$. Ocular side-effects of tolterodine and oxybutynin, a single-blind prospective randomized trial. $\mathrm{Br}$ J Clin Pharmacol. 2005;59:588-92.

30. Gatchev E, Petkova N, Braeter M, de Mey C. Ocular safety of propiverine hydrochloride in elderly patients with primary open- and narrow-angle glaucoma. Int J Clin Pharmacol Ther. 2016;54:977-86.

31. Schmidl D, Garhofer G, Schmetterer L. The complex interaction between ocular perfusion pressure and ocular blood flow - relevance for glaucoma. Exp Eye Res. 2011;93:141-55.
32. Kim M, Kim SS, Kwon HJ, Koh HJ, Lee SC. Association between choroidal thickness and ocular perfusion pressure in young, healthy subjects: enhanced depth imaging optical coherence tomography study. Invest Ophthalmol Vis Sci. 2012;53:7710-7.

Correspondence address:

Mahmut Taha Ölçücü, MD

Assistant Professor

Department of Urology

University of Health Sciences

Antalya Education and Research Hospital 07100, Muratpaşa, Antalya, Turkey

Telephone: +90 0533 512-2284

E-mail:matah_ol@hotmail.com 